\title{
Síndrome fetal alcohólico (S.F.A)
}

\author{
Dres: María Mena R.1; Eliana Selman C. ${ }^{1}$; Andrés Cantin D. ${ }^{2}$; Adriana Massardo B. ${ }^{3}$;Andrea Adriasola M. ${ }^{3}$
}

\section{FETAL ALCOHOL SYNDROME}

\begin{abstract}
Forty children with Fetal Aleohol syndrome (F. A. S.) are reported.
"There was a greater incidence of school-age children because they were refered by disturbances in learning secondary to mental retardation, $73 \%$ of them were at Specinl Sichools.

The mothers were alcoholics and most of them drank more than one titer of wine daily throughout pregnancy. $28 \%$ of mothers were dead, $23 \%$ because of Hepatic Cymosis. Their mean age at death was 32 years. $52 \%$ of Fathers were also alcoholic.

All patients showed clinical traits in accordance with those dentanded as diagnostic criterias for F. A. S.

The main symptoms were neurologic abnormalites: psychonotor development failure, difficult in learning, misbehaviour and mental retardation. Besides, there were social problems.
\end{abstract}

Reconocido es el hecho que el S. F. A. es un síndrome bastante difundido por su frecuencia, a pesar de su complejo diagnóstico. En países de Europa y en Estados Unidos desde que fue publicado en 1973 por Jones y Smith, ${ }^{1}$ se ha establecido que la frecuencia es de 1 a 2 por 1.000 R.N. con el síndrome completo y 3 a 5 por 1.000 con manifestaciones parciales. ${ }^{2}$ Sin embargo, se ha comunicado que en Estados Unidos en la costa noroeste del Pacífico en poblaciones indígenas, la incidencia del S. F. A. es de $1 \times 50$ es decir un $20 \mathrm{x}$ 1.000.3 Tambien en Boston, Massachusett, existe una elevada proporción del síndrome en la población negra. Se ha observado que el S. F. A. está estrechamente relacionado con los hábitos sociales de las poblaciones y varía según los diversos grupos étnicos y diferentes niveles socioeconómicos. Según algunos autores las frecuencias del síndrome aceptadas hasta ahora, serían sólo una mínima estimación, porque no se confiesan los hábitos de ingesta alcohólica.

Motivados por la frecuente aparición de este síndrome en la Policlínica de Genética del Servicio de Pediatría del Hospital Guillermo Grant Benavente de Concepción y como continuación de la investigación sobre S. F. A. iniciada en Valdivia en 1979,5 se decidió a recopilar y estudiar todos los casos de S. F. A. con el fin de formar una casuística importante para llamar la atención sobre este problema médico-social, ya que afecta al niño en su desarrollo pondoestatural, psiconotor

\footnotetext{
IServicio Pediatria y Sección Genetica, Hospital Guillermo Grunt Benavente, de Concepción.

2Servicio Radiología, Hospital Cuillemo Grint Benavente, de Concepuión.

${ }^{3}$ Psucúlogos Centro Diagnóstico Concepción.
}

y finalmente en su capacidad intelectual y situación social, to que justifica suficientemente su prevención.

Para diagnosticar correctamente los S. F. A. se revisó la literatura hasta encontrar una pauta que normara el diagnóstico, ya que existe un gran polimorfismo en el fenotipo, por ser un síndrome producido por un teratógeno, como el alcohol. Es asî como el Grupo de Estudio de S. F. A. de la Sociedad Investigadora de Alcoholismo determinó que el diagnóstico de $\mathrm{S}$. F. A. podría hacerse solamente cuando el paciente presenta signos en cada una de las siguientes categorías. ${ }^{3}$

1. Retardo del crecimiento pre-natal y post-natal (peso, talla y circunferencia de cráneo bajo el percentil 10) corregido para la edad gestacional.

2. Compromiso del sistema nervioso central (signos de anormalidad neurológica, retardo del desarrollo o déficit intelectual).

3. Dismorfología facial característica con al menos dos de estos tres signos:

a) Microcefalia (circunferencia de cráneo bajo el percentil 3).

b) Microftalmia y/o fisuras palpebrales cortas.

c) Escaso desarrollo del surco nasogeniano, labio superior fino $y / o$ aplanamiento del área del maxilar.

\section{MATERIAL Y METODO}

En la Policlínica de Genética del Servicio de Pediatría del Hospital Guillermo Grant Benavente, de Concepción, $y$ en un periodo de 18 meses, comprendido entre marzo de 1980 y septiembre de 1981 , se estudiaron 54 niños con diagnóstico presuntivo de S.F.A. Estos nin̄os procedían de Concepción y de otras ciudades de la Octava Re- 
gión y de diferentes fuentes de referencia: hogares de menores en situación irregular, aldeas, Conin, colocaciones familiares, Policlínica de Pediatria y Endocrinología y Servicio de Pediatría, especialmente de la Unidad de Lactantes y R.N., la mayoría de estos niños eran referidos por retardo pondoestatural o psicomotor y/o retardo mental o por sospecha de genopatia.

A todos los pacientes con antecedentes y rasgos compatibles con S.F.A. se les completó una ficha, previamente confeccionada y que contenía los datos anamnésicos y signos clinicos más destacados del síndrome. Los antecedentes familiares en varios casos clínicos fueron aportados por familiares, asistentes sociales o personal de hogares infantiles, etc., ya que algunas madres habían fallecido, por lo que los antecedentes personales y lamiliares están incompletos.

Otros niños que presentaban rasgos clínicos y neurológicos compatibles con S.F.A. no se pudieron diagnosticar como tales, principalmente por dos rizones: l. Por no existir antecedentes de a]coholismo materno al ser abandonados en hospitales u otras instituciones sociales. 2. Por negar las madres ingestión de alcohol durante el embarazo o referir volúmenes aparentemente insuficientes para provocar el síndrome. ${ }^{6}$ Quedando todos estos niños en control para su seguimiento y posible esclarecirriento diagnóstico.

Con la norma para diagnóstico de S.F.A. de la Sociedad Investigadora de Alcoholismo se revisaron las fichas clínicas y se descartaron aguellas que no cumplían con el mínimo de rasgos clínicos exigidos. Estos niños quedaron calificados como "Hijo de madre alcuhólica" o "Efectos tetales del alcohol", para sus controles posteriores. Finalmente quedaron evaluados como S.F.A. 40 niños, a todos ellos se les solicitó examen de orina, radiografia de cráneo y carpo y test psicométrico, éste se efectuó a los escolares en el Centro Diagnóstico, se empleó la Escala de Inteligencia de Wechler para nin̄os (Wisc); solamente en 4 menores de 7 años se efectuó evaluación psicométrica, por no haber psicólogo en el Servicio de Salud. En tres lactantes se empleó la Escala de Evaluación del Desarrollo Psicomotor (EEDP) de Soledad Fodríguez y cols., y en un preescolar el Test de Terman M.

\section{RESULTADOS}

El mayor número de pacientes con S.F.A. era de Concepción $=16$; luego Talcahuano $=6$; Coronel
$=4$; Lota $=3$; de Chiguayante, Chillán, Florida y Tomé procedían los pacientes de cada ciudad y de Santa Juana l. La mayor incidencia por grupo etario fue para los escolares $=15$; lactantes $=11$; preescolares $=9$, y recién nacidos $=5$ (Fig. 1). De los 15 escolares, 11 estaban en escuela especial (73\%). La ubicación actual de los 40 niños es la siguiente: 12 con su madre, 12 con otros familiares, 6 en hogares infantiles o colocaciones familiares, 6 adoptados y 3 en aldeas (Fig. 2).

\section{Figura 1}

FRECUENCIA POR GRUPO ETARIO OE 40 S.FA. HOSP. G GRANT B. CONCEP.

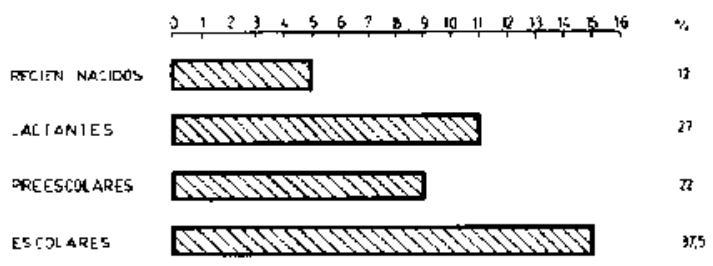

Figura 2

UBICACION ACTUAL DE 40 NIÑOS CON SFA. HOSP. G. GRANT B. CONCEPCION

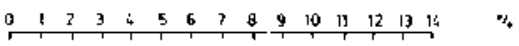

HDGAR PAIERNO

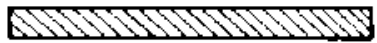

30

HOGAR FAMIL AH

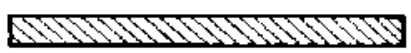

32

HOGAR INFANTI

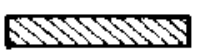

4

A DOPIAJUS

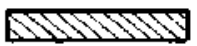

15

A. JEAS

बाप

7.5

Antecedentes matemos: El término medio de las edades conocidas fue de 34 años, 11 madres habían fallecido, 9 tenían como causa de muerte el diagnóstico de cirrosis hepática, en dos madres este diagnóstico no fure precisado, pero eran alcohólicas antiguas. El término medio de la edad de fallecimiento por cirrosis fue de 32 años.

La modalidad y tiempo de ingestión del alcohol fue el antecedente más difícil de obtener, sólo en 8 casos se supo la antigüedad del alcoholismo y generalmente en forma indirecta; resultaron 4 grupos de 2 madres en que la duración del alcoholismo era de 5, 10, 15 y 20 años. Veintiséis madres ingirieron alcohol durante todo el embarazo. Bebieron más de 1 litro de vino casi diariamente = $14(53 \%)$; más de 2 litros $=4$, y más de 5 litros 1 embarazada. Dos madres declararon haber bebido sólo los 4 primeros meses del embarazo, sin 
embargo, uno de sus hijos fue uno de los S.A.F. más severos, con cardiopatía congénita.

En las historias clínicas, a pesar de ser incompletas, se detectó aumento del número de abortos. Cuatro abortos en un total de 5 embarazos; 3 de 12; 4 de $14 ; 2$ de $3 ; 6$ de $12, y 3$ de 8 .

En cuanto a los hijos fallecidos, en una familia hubo 8 fallecidos de un total de 12 hijos.

Antecedentes paternos: Veintiún padres eran alcohólicos $(52 \%)$ y $\mathbf{5}$ fallecidos; se ignora causa. Un solo padre era rehabilitado y también una madre con 2 niños con S.F.A. (Tabla 1).

\section{Tabla 1}

Situación actual de 39 padres de 40 S.F.A. Hospital Guillermo Grant Benatvente, Concepción.

\begin{tabular}{lcc}
\hline & Madres & Padres \\
\hline Alcoholicos & 26 & 21 \\
No alcoholicos & 2 & 12 \\
Fallecidos & 11 & 5 \\
Por cirrosis & $(9)$ & $(?)$ \\
Hehabilitados & 1 & 1 \\
\hline
\end{tabular}

Desarrollo prenatal: Los pesos de nacimiento se conocieron sólo en 27 niños y en $22(81 \%$ ) fue menor de 2.500 gramos (Fig. 3).

Figura 3

DESARROLLO PRENATAL OE 27 S.F.A HOSP. G GRANT B. CONCEP.

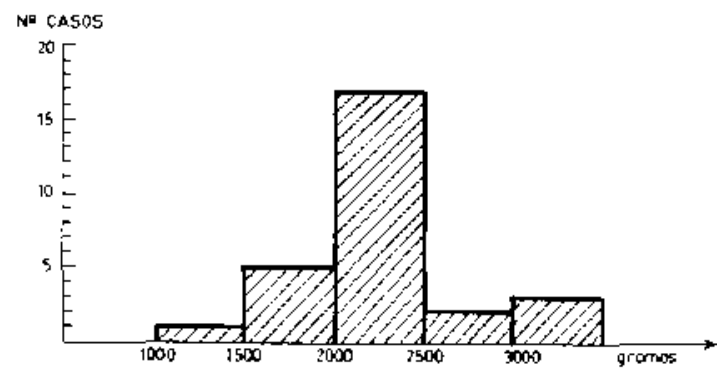

En 3 R.N. se observó temblores, hiperexcitabilidad, rubicundez, en ellos se descartó hipoglicemia e hipocalcemia, posteriormente se interpretaron como signos de deprivación alcohólica.

Desartollo postnatal: La talla estaba bajo el percentil 3 en el $87 \%$ de los pacientes y en el $90 \%$ se encontró peso y C.C., tambiên bajo el percentil 3.

Compromiso de SNC: La alteración más frecuente fue retardo del desarrollo psicomotor en $38(90 \%)$, hiperkinesia en $21(52 \%)$, por este síntoma varios niños estaban en tratamiento neurológico.
Los test psicométricos de los 9 escolares dieron como resultado CI que fluctuaron entre 50 y 75 $(50,53,54,62,61,67,70,70,75)$ término medio 62 .

En los lactantes los CI fueron: 0,40-0,50-0,75, lo que equivale a 2 retrasos psicomotores $y$ un riesgo psicomotor. En el preescolar el CI fue de 0,90 o desarrollo mental nomal.

Las alteraciones craneofaciales más frecuentes fueron las exigidas en la clasificación del síndrome como microcefalia, blefarofimosis, etc. (Figs. 4 y 5), y aparecen en la letra A de la Tabla 2. En la letra $B$ está el resto de los signos menos frecuentes. Entre éstos destaca por su importancia la cardiopatía congénita que se encontró en 5 niños, en 3 de ellos no representa actualmente problema, porque la signología cardíaca desapareció alrededor de los 6 meses de edad. Sólo en dos adquirió signos de gravedad; a un lactante con Tetralogia de Fallot se le practicó operación de Blalock, y el otro tiene un defecto septal ventricular e insuficiencia cardíaca (Fig. 5).

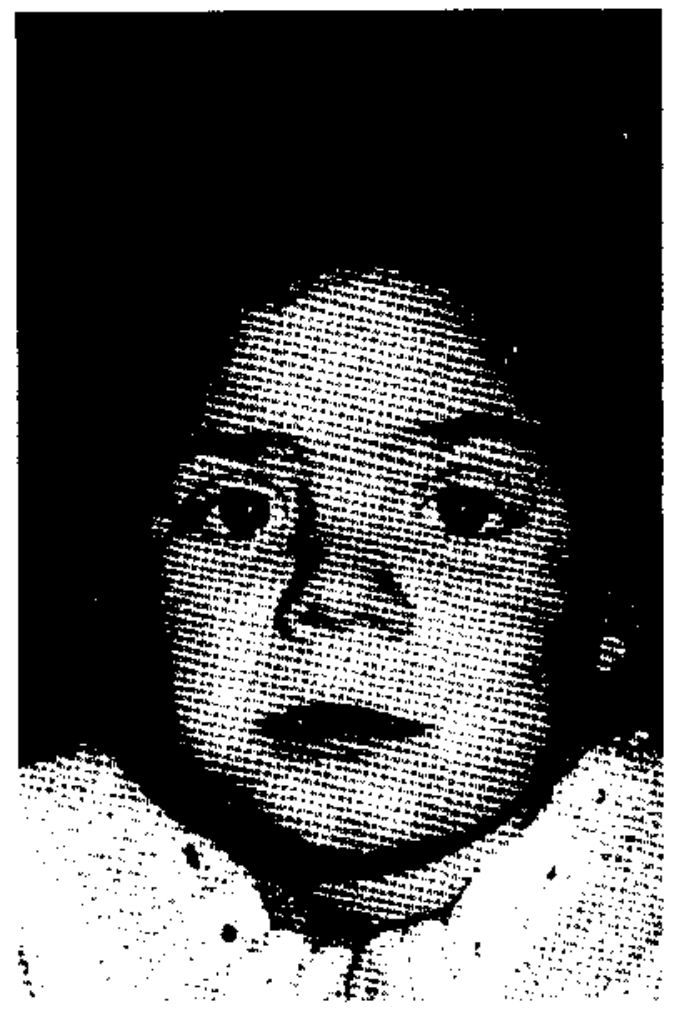

Figura 4. S.F.A., 5 años de edad, se observa: microcefalian, bletarofimosis, ansencia de surco nasogeniano, lit bio superior fino. 


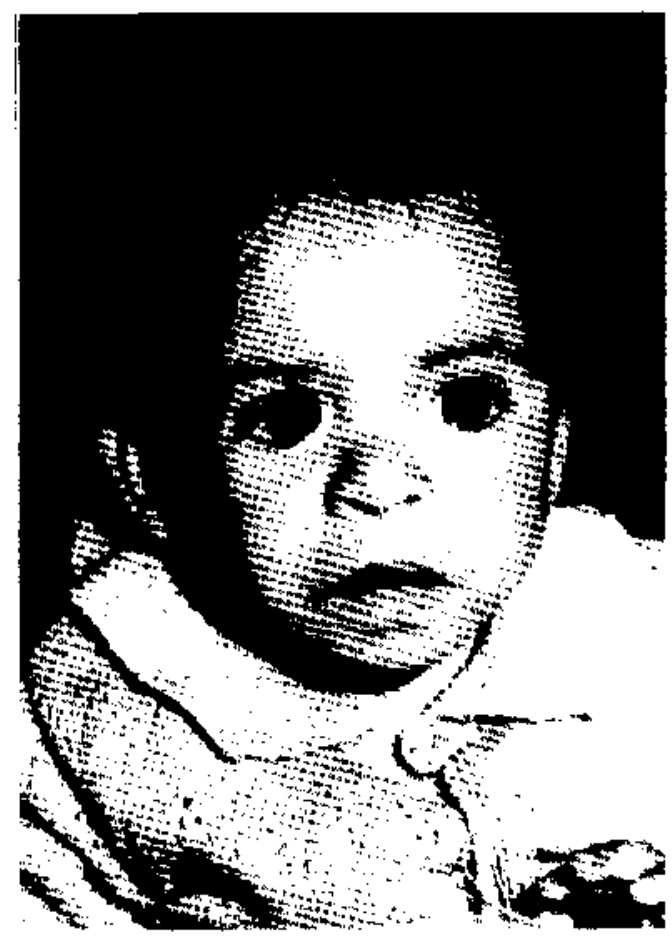

Figura 5. Lactante de 9 meses con S.F.A. destaca: microcefalia, blefarofimosis, epicanto, ptosis palpebral derecha, hipoplasia medio facial, labio superior fino.

Tabla 2

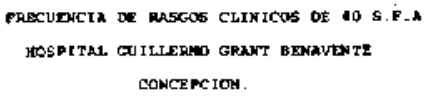

A. PUSGOS CLINICOS mas FAREUENTES.

\begin{tabular}{|c|c|c|c|}
\hline 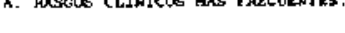 & & Ue Canos & - \\
\hline Petar do del desurtollis preatal & $c 25009 \mathrm{~g}$. & 22 de 27 & 11 \\
\hline Metordo del deeorzolio poetnatal, & Talle $<P_{3}$ & 35 d* 40 & a) \\
\hline & $P=0<P_{g}$ & 36 de 40 & 90 \\
\hline Petardo mextal & & 12 de 13 & $\$ 2$ \\
\hline Hetardo del desarrollo poscomotor & & $3 B$ de 10 & 95 \\
\hline MICHOFEFALIA & $* \mathbf{P}_{\mathbf{3}}$ & 36 de 10 & 90 \\
\hline BLEFNROP TKOSIS & & 34 & 65 \\
\hline UIPOPLASIA MEDIO FACTAL. & & 27 & 67.5 \\
\hline AJSEMCIA OE SUPCO NASOCEETAHO & & 30 & 75 \\
\hline LNAO SCPEFIOR FINO & & 32 & 9o \\
\hline
\end{tabular}

B. RASCOE CLINICOS AENCS PRECLENTES

HIPERKIIESIA

IWCOORDINRCIDN MOTORA

QIPOTONIA

TEVIOARS

ptosis paLPEeral

EFICAETO

ESTRAB TSHO

HIPERTELORTSMO

MTCROGKNSIA

BRDGMATISW MELATINO

phlador ojivac.

ORENAS SIMPLES

LAETHA BRTRS

CARDDOEATIA CONGENITA

BIPERTEL IA

H IPOPLAS]A GENITALES mascal INOS

MIRSUT ISVO

HQunacions

HIPOQLESTA ULTLAA FALNAE

hIPOPLASTA uras

OPAGTDMD CORNEAL
Las líneas palmares aberrantes se observan en $35 \%$, las más frecuentemente encontradas fueron línea pseudosimiana, simiana y 5 con sólo 2 líneas palmares que se consideran patognomónicas del síndrome.

Entre las alteraciones poco frecuentes se observó opacidad corneal bilateral en 1 recién nacido.

Las radiografias de cráneo practicadas en 16 nin̄os revelan en un $100 \%$ microcefalia, sólo un cráneo presenta aumento notorio de las huellas de las circunvoluciones.

La edad ósea estaba retrasada en los niños que además tenían desnutrición primaria y como una observación poco frecuente hubo sinostosis radiocubital en un paciente. ${ }^{7}$ Las pielografias de eliminación fueron normales en 3 pacientes en que se solicitó por presentar G.N.D.A. No se practicó este examen en los otros niños, por ser normales todos los exámenes de orina y ser además un examen de al to costo.

\section{COMENTARIO}

La mayoría de los pacientes con S.F.A. eran de Concepción, pero un número importante de niños procedía de otras ciudades; Lota y Coronel, por ser zona minera, tienen un al to índice de alcoholismo, como también lo tienen potencialmente Chillán, Florida y Quillón, por ser zona vitivinícola.

E] mayor número de niños con el síndrome eran escolares, edad en que se manifiestan las dificultades de aprendizaje, es asi como el $73 \%$ estaban en escuela especial y el resto tenía mal rendimiento escolar.

Debe destacarse que hubo varios escolares hijos de madre alcohólica con dificultades de aprendizaje y que sin embargo no mostraban los estigmas completos del síndrome, lo que está de acuerdo con observaciones respecto a que el S.F.A. completo esté alejado al final del espectro del problema fetal causado por ingestión de alcohol durante el embarazo y que existen muchos rasgos patológicos no específicos en estos niños. En ausencia de algunas de las características necesarias para diagnosticar el síndrome puede usarse el término "Efectos Fetales del Alcohol" (F.A.E.).

También se refiere en la literatura que de madres bebedoras excesivas se observaron R.N. sin anomalias físicas, pero que mostraban disturbios funcionales como alteración de la regulación del sueño, demostrada con EEG y que podían pers is- 
tir durante meses. Experimentos en animales han revelado alteraciones del EEG en fetos durante infusión materna de alcohol. ${ }^{8}$

El alcohol usado en dosis terapéuticas antes de partos prematuros, con el propósito de iniciar la maduración de las enzimas hepáticas del feto, ha provocado en los R.N. depresión del S.N.C., acidosis, disminución de la frecuencia respiratoria, Apgar bajo, anomalias de la médula ósea, etc. ${ }^{9}$ Semejantes alteraciones se producen en las intoxicaciones alcohólicas durante el embarazo, produciéndose anomalías de los óryanos que están en desarrollo y con una acelerada división celular, variando estas anomalias con las diversas características de la ingesta alcohólica en cantidad y duración, pero especialmente importante es su precocidad en el embarazo. ${ }^{10}$

De la misma forma se producen las alteraciones del sistema nervioso central, que se manifiestan posteriomente como disfunciones cerebrales mínimas, dificultad de aprendizaje, alteraciones conductuales, disturbios del sueño, etc. La gama de lesiones neurológicas es amplia y se observa desde alteraciones bioquimicas solamente hasta microdisplasias, hidrocefalian agenasia del cuerpo calloso, porencefalia, arrinencefalia, etc. ${ }^{9}$ También se ha observado que el problema de la dismorfogénesis cerebral puede ocurrir como efecto predominante en la exposición uterina al alcohol, lo que significa que en algunos nin̄os el problema de estructura $y / 0$ función cerebral puede ocurrir como única anormalidad aparente. ${ }^{\text {" }} \mathrm{El}$ mecanismo de acción del alcohol permanece desconocido, algunos investigadores refieren que el etanol es teratógeno por sí mismo o su metabolito el acetaldehído, otros consideran que el defecto nutricional asociado juega un papel importante ${ }^{12} y$ también el déficit de zinc. ${ }^{10-13}$

El hecho de que 5 de los S.F.A. hayan sido diagnosticados como R.N. tiene importancia, porque significa que los pediatras están conscientes del problema y se busca, o' que la frecuencia del síndrome va en aumento. Incluso se observaron signos de deprivación alcohólica en algunos de los R.N.

En referencia a los antecedentes matemos, el término medio de la edad materna avanzada, 34 años, porque frecuentemente los hijos con S.F.A. son los menores y nacen en edades entre 30 y 35 años, cuando el alcoholismo está establecido y avanzado, pues se ha observado que empieza alrededor de los 20 años. ${ }^{4}$ Sin embargo, la edad media matema de fallecimiento por cirrosis hepática fue de 32 años, Jo que indica que el alcoholismo cono enfermedad es mucho más intensa y corta en las mujeres que en los varones. Esto se debe a la modalidad de ingestión alcohblica de éstas, que beben solas, en dosis bajas pero frecuentemente. En cuanto a la incidencia de cirrosis hepática por alcoholismo en la mujer, tenemos el antecedente que en el Hospital Guillermo Grant Benavente del total de autopsias por cirrosis hepática el $36 \%$ corresponde al sexo femenino. ${ }^{14}$

En las historias maternas se pudo observar que la mayoria de las madres bebian más de un litro de vino al día, lo que equivale a $90 \mathrm{cc}$ de alcohol puro, cifra que se ha considerado de mayor riesgo para el feto, ${ }^{2}$ pero volúmenes de 30 a $60 \mathrm{cc}$ diarios también han mostrado alteraciones fetales. ${ }^{15}$ Dos madres refirieron haber bebido exageradamente sólo los 4 primeros meses de embarazo; sin embargo, uno de los hijos tenia un S.F.A. severo. Ante estos hechos se pueden plantear dos interrogantes: ¿Fue fidedigno el antecedente materno? ¿Basta la ingestión alcohólica excesiva durante los primeros meses de embarazo para producir el sindrome ?10 También se observó una tendencia al aumento de los abortos y de la mortalidad infantil. $^{2}$

El 52\% de los padres del goupo de estudio era alcohólico. Si consideramos que Lemoin ${ }^{16}$ publicó que en una tercera parte de un grupo de 127 S.F.A. solamente el padre era alcohólico, explicundo el mecanismo de acción por alteración de las células germinales, el problema en nuestro país se agrava, dados ios altos índices de alcoholismo prevalentes.

Respecto a las caracteristicas clínicas de los $\mathbf{4 0}$ niños estudiados, en el $80 \%$ de ellos el peso de nacimiento fue inferior a 2.500 gramos. Algunos autores comunicaron que no tan sólo la ingesta de alcohol durante el embarazo posee un riesgo de crecimiento fetal, también una historia de alcoholismo materno lo biene, independientemente del alcohol ingerido en el nuevo embarazo. ${ }^{17}$

El desarrollo postnatal en el $87 \%$ de los niños estaba bajo el percentil 3, pero esto se observa sólo en los primeros años de vida, porque en la edad escolar los parámetros peso y talla van mejorando hasta llegar a valores generalmente normales bajos. ${ }^{16}$

Los rasgos craneofaciales más importantes exjgidos en la clasificación de S.F.A. tenían elevada frecuencia, además del retardo del desarrollo pre 
y postnatal y aiteraciones neurológicas, ya que aislados ninguno de los 3 parámetros tiene valor diagnóstico.

Respecto a otras malformaciones, la cardiopatía congénita se presentó en un $12 \%$ de los niños, lo que es bajo con respecto a otras series que refieren un $30 \%,{ }^{18}$ otros un $49 \%$. $^{19}$

Las malformaciones cardíacas más frecuentes son defectos septales, displasia de la arteria pulmonar ${ }^{20} \mathrm{e}$ interrupción del arco aórtico tipo $\mathrm{A}$ con comunicación aortopulmonar. ${ }^{21}$

Entre las alteraciones poco frecuentes se observó opacidad comeal bilateral, aparece reportado en la literatura en un S.F.A. junto con defecto del tubo neural y anomalía renal. ${ }^{22}$

Sin embargo, el mayor problema para todos estos nin̄os lo constituyen las alteraciones neurológicas, que interfieren en su vida de relación tanto en el hogar como en la escuela por el retardo psicomotor, como marcha y lenguaje retardado, ${ }^{23}$ gran hiperkinesia, alteraciones conductuales, como tugas de hogares adoptivos y en edad escolar incapacidad de aprender al ritmo de los niños normales, demostrándose retardo mental en un elevado porcentaje $y$ con témino medio de CI más bajo que en otras series. ${ }^{24}$

Actualmente en Estados Unidos el S.F.A. es reconocido como la tercera alteración más frecuente en la que el retardo mental es un componente ${ }^{25}$ y la primera causa de retardo mental absolutamente prevenible. ${ }^{12}$ Se sugiere que la manifestación más sutil de la disfunción del sistema nervioso central son los trastornos conductuales y de aprendizaje en niños expuestos al alcohol en el útero, por lo que deberían ser seguidos cuidadosamente en los primeros años de escolaridad parat detectar estos sintomas.

Se ha demostrado que hijos de madres alcohólicas con signos de S.F.A. y con inteligencia normal estaban en colegios especiales por trastornos del aprendizaje $y$ conductuales. ${ }^{25}$

El otro gran problema de estos niños con S.F.A. es el social, ${ }^{10}$ porque en la mayoría de ellos además de la deficiente situación socioeconómica familiar, agudizada por el alcoholismo, se observó una desintegración familiar progresiva. Es por esto que sólo un $30 \%$ de los niños vivían en su hogar y eran los R.N. y lactantes, todos ellos estaban en las peores condiciones generales, y el 70\% restante estaba repartido en hogares de familiares a infantiles, aldeas y adoptados. Estos últimos tienen actualmente múltiples problemas familia- res, porque las madres adoptivas, a pesar de que comocian los antecedentes alcohólicos matemos, ignoraban que pudiera ser la causa de la hiperkinesia, retardo mental o alteraciones conductuales de estos niños.

A pesar de que el alcohol está reconocido como agente embriotóxico y que se reconoce mundialmente el aumento del alcoholismo, habría un escaso número de niños afectados, la discrepancia se debe a varias razones: 1 . Desconocimiento del antecedente de alcoholismo en embarazadas. 2 . Falta de relación entre alcoholismo matemo $y$ daño fetal, que no siempre está presente. 3 . No se sabe con qué frecuencia este riesgo conduce a manifestaciones, porque algunos cambios pueden ser variaciones normales de retardo mental, 4 . El efecto de consumo de alcohol en el embarazo precoz tiene un alto riesgo y un amplio espectro, que va desde malformaciones letales a una moderada o escasa disminución de la inteligencia. ${ }^{10}$

Por todo lo anteriormente expuesto, consideramos que es de la mayor importancia difundir estos conceptos primero entre los pediatras, obstetras y matronas para poder diagnosticar y prevenir el sindrome. Se ha podido observar que la abstinencia de alcohol, aunque sea en el segundo o tercer trimestre del embarazo, tiene ventajas maternoinfantiles con respecto a las que lo inginieron durante todo el embarazo. ${ }^{26}$

Además, considerando que el S.F.A. tiene una frecuencia bastante importante al menos en la Octava Región, se debería difundir estos conceptos con charlas educativas a la población, porque de esta manera las madres bebedoras podrían elegir entre limitar definitivamente su descendencia o abstenerse del alcohol para poder procrear conscientemente.

De todas maneras se continuará la investigación sobre S.F.A. contabilizando los nuevos casos y tambièn yendo a buscarlos a los lıgares de los que provenían más frecuentemente, como hogares de menores y escuelas especiales.

\section{AGRADECIMIENTOS}

Se agradece al personal y Director del Centro Diagnóstico, Sr. Benedicto Salgado, por la colaboración prestada a este estudio.

\section{REFERENCLAS}

I Jones, K. L.; Smith, D. "Patterns of inalfomations in offspring of thronic alcoholic enothers". Lancet, I: 1267-1271, 1973. 
2 Sterling H.C. "The fetal alcohol symdronue", Euglaud. J. Med. 248: 1963-1967, 1978.

3 Roset, H. L.; Weiner, L. "Strategies for prevencion of fetal aucohol effects". Ohstetrics and Gynecology, vol. 57 (1): 1-7, Jan. 1981.

4 Erb, L.; Andersen, B. D. "The tetal al (cohol syndrome". (F.S.A.) A review of the impact of chronic matemal alcoholism on the develuping letus. Clin, Pediatr. (PHILA) 17 (8): 644-649, Aug. 1978.

5 Mena, M.; Albomo, C. "Sindrume fétal aleohólico. Estudio de 19 casos clínicos". Rev. Chil. Pediatria 51 (16): 414-423, now. dic. 1980.

6 Lipson, A.; Yen, J. "Alcohol and phenilketonuria". Lanext (1) 8222: 717-718, March 1981.

7 Rosche, $E$ "Skeletal defects and fetal alcohol syndrome". Arch. Internac. Med. 139 (9): 959, Sept 1979.

8 Mann, C. "Eiffect of alcohol on fetal cerebral function and metabolism", An. J. Obstetries and Gynecology, Vol. 122 (7): 84.5, Aug. 1975.

9 Wisnicusid, K.; Lopm, R. "F.A.S. and related C.N.S. problems". Neurology 29 (10): 1429-1430, Oct. 1979.

to Hinkers, $H$. J. "The inlluences of' alcohol on the fetus". J. Perinat Med, 6(1): 3-14, 1978.

11 Stering, C.; Elsworth, A "Brain malfonnation related to prenatal exposure to ethanol". J. of Pediatr. 92: 61-187, 1978.

12 Beatie, J. "Alcohol and the tetal brairr". Lancet (1) B223: 788, atsril I:981.

13 Fiymn, A.; Matier, S. "Zine status pregnant idcoholic women: a determinant of fetal outerme". Lancet 8220 (1): 572-574, March 1981.

14 Shumann, $R$. "Alcoholismo y cirros is nutricional". Apartado del boletin de la Sociedad de Biología de Concención, Tomo XIII: 3-13, 1970.
15 Hanson, d. W.; Streissguth, A. P.; Smith, D.W. "The eflects of moderate alcohol consumption during pregnancy on letal growth and morphogenesis". J. Pediatr. 92 (3) 457-460, March $197 \mathrm{y}$

16 Lemoine, P.; Haronsseau, H. L. "I.es enfarts de parents ilcroo liques. Anomalies abservies a propos de 127 cas". Quest Med. $25 ; 476,1468$.

17 Little, R. E; Streissguth, A P. "Decreased bitth weigh in infant of alcobolic women who abstaines during pregnancy". J. Ped. $96\{6\}$ : 974-977, June 1930 .

16 Polmer, $R$. "Congenital malfomations in offspring of thronic alculiolic riother". Pediatrie 53: 490194, 1974.

19 Hanson, $d_{\text {; }}$ Woolf, P. "Cardiovascular maltonnations in the fetal aleohol syndrome. Experience with $4 \perp$ patients". J.A. M.A. 235 (14): 1454-1460, 1976.

20 Steeg, C.; Woolf, P. "Cardiovascular malformations in the fetal alcohol syndrume". Am. Heath J. 98 (5): 635- +237 , Now. 1979 .

21 Temapon, M.: Schreider, $P$. "Aortic arch interrupton type н with aortopulmortary fenestration in an offsprings of chronic alcuholic mother". Helv. Poediatr. Acta :32 (2): (4)-148, Jul. 1977 .

22 Goldstein, G.; "Neurd tube defect and renal anomalies in a Child with F.A.S.". J. r'ediatr. 93 (4): 636-637, Oct. 1978.

23 losub, S.; Fuchs, $M$. Felal alcohol Symdrome Revisited Pediatrics, Vol. 68: 475-479, Ott. 1981 .

24 Steissguth, A P.; Herman, C. S. "Intelligence, bebaviour and dismomphogenesis in the fetal alcorhol symdrume". J. Pediatr. 92: 363, 1978 .

25 Shaywitz $S_{\text {; }}$ Cahen, $A$ "Behaviour and learning difficultoes in children of normal iritelligence born to alcohulic mothers". J. Yediatr. 96 6): 978-982, June I\$80.

26 Rosett, H.; Ovelletse, E. "I'erapy of heavy drinking during pregnaucy". Obstetrics and Gytocology, Vol. 51 (1): 41-46. Ja1n. 1978. 